\title{
Editorial
}

http://dx.doi.org/10.14483/udistrital.jour.calj.2015.2.a00

\section{Belonging to a Community of Research Practice}

Belonging to a community of research practice as applied linguists or as academics in any field is part of our professional life. Being an academic implies, inter alia, creativity in advancing knowledge in the disciplines, which reflects in writing journal articles, presenting papers in conferences, doing research, teaching, tutoring students and publishing. Globally, every higher education institution requires that academics publish in prominent journals to make their work and their institution visible and influence their professional field. However, the questions that arise concerning academic production are how do communities of research support academic production?, How do higher education institutions help novice researchers develop academic writing competences?, What is the place of writing within research? How do institutions foster quality publication?

Rowena Murray (2012) shared similar concerns about the writing and publishing dimensions of research productivity and how writers' understanding of the nature of journal article writing can be developed. She believes that although the imperative to publish is widely accepted, we need to know more about the process by which it is achieved. She claims that higher education institutions assume that novice researchers produce fewer publications than experienced researchers do, but there has been no exploration of how their writing practices develop. Finally, she proposes that in order to learn about academic writing, we can listen to academics ${ }^{\prime}$ accounts of how they work to meet research assessment targets.

Being a journal editor myself, my experience includes mentoring authors who struggle to have the manuscript submitted for publication approved. It also has to do with explaining novice and experienced researchers the subtleties of submitting the article. In my dual role as editor and academic writing instructor, aware of the challenges of writing to publish, I decided to offer an 11-session writing for publication course every two years from 2009-2013 in order to establish an academic community that supported the writing process of many researchers interested in publishing. The outcomes of the courses report thirteen articles published, 10 in national and 3 in international journals and the valuable writing experience for the participants and for me as instructor. Some of the reflections from the participants are having to think about their audience, getting to know the structure of the article for the journal selected, and carefully selecting the academic language required (Clavijo, 2014).

In addition to the above aspects, writing journal articles implies knowing and following the code of ethics for research and for publications. Novice researchers might not be aware of the ethical regulations in academic journals. The Colombian Applied Linguistics journal is inviting researchers and authors to know its code of ethics for best publication practices (Declaración de buenas prácticas editoriales y 
normas éticas) that is included in this issue for the first time (p. 325-326). Our institution as a member of the Committee on Publication Ethics (COPE) gives emphasis to ethical practices.

I propose to develop communities of writing practice by means of communities of research practice at our higher education institutions. Communities of practice, as Wenger (1998) puts it, are groups of people who share a concern or a passion for something they do and learn how to do it better as they interact regularly. Thus, if we work on creating healthy productive environments within our communities of research in higher education institutions we should possibly facilitate and successfully develop our research and publication agendas.

\section{References}

Clavijo, A. (2014). La escritura científica y la producción de conocimiento con profesores universitarios. Presentación en el Primer Simposio sobre Formación Pedagógica y Didáctica de Docentes Universitarios. Universidad Distrital. Bogotá.

Murray, R. (2012). Developing a community of research practice. British Educational Research Journal. 38, 5, ppp.783-800.

Wenger, E. (1998). Communities of practice: learning meaning and identity. Cambridge: CUP.

\section{Amparo Clavijo-Olarte}

Editor 


\section{Editorial}

\section{Pertenecer a una Comunidad de Práctica de la Investigación}

Pertenecer a una comunidad de investigación y de práctica como académico en el área de la lingüística aplicada o en cualquier otro campo es parte de nuestra vida profesional. Ser un académico implica, entre otras cosas, la creatividad en el desarrollo del conocimiento en las disciplinas, el cual se ve reflejado en la escritura de artículos para revistas, presentación de documentos en conferencias, realización de proyectos de investigación, la enseñanza, tutoría a estudiantes y la publicación. A nivel mundial, cada institución de educación superior requiere que los académicos publiquen en revistas prominentes para hacer su trabajo y el de su institución visible e influir en su campo profesional. Sin embargo, las preguntas que surgen en relación con la producción académica son ¿Cómo las comunidades de investigación apoyan la producción académica? , ¿Cómo las instituciones de la educación superior ayudan a los investigadores novatos a desarrollar las competencias en la escritura académica?, ¿Cuál es el lugar de la escritura dentro de una investigación?, ¿Cómo las instituciones promueven la calidad de la publicación?

Rowena Murray (2012) comparte preocupaciones similares sobre la escritura y publicación como dimensiones de la productividad en la investigación y sobre la necesidad que los autores comprendan acerca de la naturaleza de la escritura de un artículo científico. Ella cree que aunque la obligación de publicar es ampliamente aceptada, necesitamos saber más acerca del proceso que implica lograrlo. Ella asegura que las instituciones de educación superior asumen que los investigadores novatos producen menos publicaciones que los investigadores experimentados, pero no se ha explorado cómo se desarrollan sus prácticas de escritura. Por último, ella propone que, a fin de aprender acerca de la escritura académica, podemos escuchar de casos académicos acerca de cómo los investigadores trabajan para cumplir las metas en la evaluación de los productos de investigación.

Siendo yo misma editora de una revista, mi experiencia incluye apoyar a los autores que tienen dificultades para preparar un manuscrito y lograr que su publicación sea aprobada. Mi tarea también implica guiar a investigadores novatos y experimentados sobre las sutilezas de presentar y enviar un artículo para evaluación. En mi doble papel como editora e instructora de la escritura académica, consciente de los retos de escribir para publicar, decidí crear un curso de 11 sesiones sobre escritura académica que se ofreció cada dos años a partir de 2009 y hasta el 2013 con el fin de establecer una comunidad académica que apoyara el proceso de escritura de varios investigadores interesados en publicar. Los resultados de los cursos reportan trece artículos publicados, diez en revistas nacionales, tres en revistas internacionales y la valiosa experiencia de la escritura para los participantes y para mí como instructora. Algunas de las reflexiones de los participantes incluyen pensar en la audiencia que va a leer el artículo, aprender sobre el formato y la estructura del artículo que requiere la revista específica donde se quiere publicar, y seleccionar cuidadosamente el lenguaje académico (Clavijo, 2014). 
Además de los aspectos anteriores, la redacción de los artículos implica conocer y seguir el código de ética para la investigación y para la publicación. Investigadores novatos podrían no estar al tanto de las regulaciones éticas en las revistas académicas. La revista Colombian Applied Linguistics está invitando a investigadores y autores a conocer su código de ética para mejores prácticas de publicación (Declaración de Buenas Prácticas editoriales y Normas éticas) que es incluido en esta edición por primera vez (pág. 325-326). Nuestra institución como miembro del Comité de Ética en Publicacones (COPE) hace énfasis en prácticas éticas de publicación.

En efecto, propongo desarrollar comunidades de prácticas de la escritura por medio de comunidades de práctica de la investigación en nuestras instituciones de educación superior. Las comunidades de práctica, como Wenger (1998) lo dice, son grupos de gente quienes comparten una preocupación o una pasión por algo que ellos hacen y aprenden cómo hacerlo mejor, ya que interactúan regularmente. Por lo tanto, si trabajamos en la creación de ambientes productivos sanos dentro en nuestras comunidades de investigación en la educación superior, nosotros deberíamos posiblemente facilitar y desarrollar con éxito nuestras agendas de investigación y publicación.

\section{Referencias}

Clavijo, A. (2014). La escritura científica y la producción de conocimiento con profesores universitarios. Presentación en el Primer Simposio sobre Formación Pedagógica y Didáctica de Docentes Universitarios. Universidad Distrital. Bogotá.

Murray, R. (2012). Developing a community of research practice. British Educational Research Journal. 38, 5, ppp.783-800.

Wenger, E. (1998). Communities of practice: learning meaning and identity. Cambridge: CUP.

Amparo Clavijo-Olarte PhD

Editora 